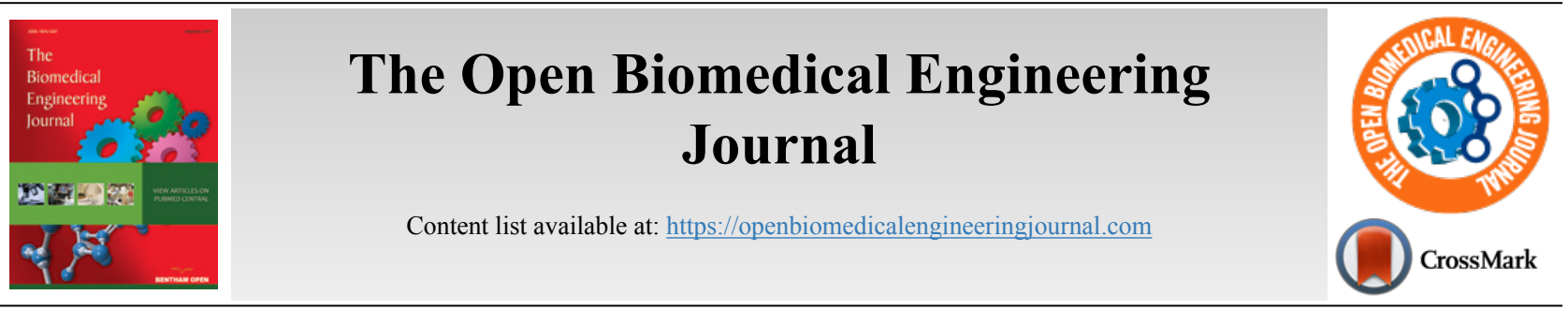

REVIEW ARTICLE

\title{
Telemedicine and Teleconsulting in the Era of COVID-19 Pandemic: A Useful Tool from Screening to Intensive Care Monitoring
}

\author{
Raffaele Abete ${ }^{1, *}$, Andrea Lorenzo Vecchi ${ }^{2}$, Attilio Iacovoni ${ }^{1}$, Andrea Mortara ${ }^{3}$ and Michele Senni ${ }^{1}$ \\ ${ }^{1}$ Cardiovascular Department, ASST Papa Giovanni XXIII, Bergamo, Italy \\ ${ }^{2}$ Department of Heart and Vessels, University of Insubria, Varese, Italy \\ ${ }^{3}$ Cardiology Department, Policlinico di Monza, Monza, Italy
}

\begin{abstract}
:
The COVID-19 global pandemic has had striking effects on clinical practice and medical assistance and the progressive evolution of telemedicine and telehealth systems has allowed healthcare professionals to connect with patients yet respecting the striking need for social distancing. This article aims to review the possible ways to use telehealth and teleconsulting systems to guarantee an adequate level of clinical assistance starting from screening procedures up to support the management of patients admitted to intensive care units area, thus balancing the need to ensure continuity of care and at the same time limiting the possible sources of contagion expansion. Telemedicine may be a useful tool to improve clinical assistance and reduce the financial burden on the health system in a long-term view. Although it cannot completely replace patient-physician interactions, it would be desirable to implement this field and made it accessible to the largest part of the population.
\end{abstract}

Keywords: Telemedicine, Telehealth, Teleconsulting, COVID-19, Screening, Intensive care monitoring.

\section{INTRODUCTION}

Telemedicine was initially designed in order to reach the greatest number of patients and optimize the care delivery system and financial viability, especially in emergency situation, like natural disasters or any critical scenario, which makes the care delivery process difficult when critical infrastructures are compromised [1]. From the first decade of the century, telehealth has progressively started to include everyday clinical practice, integrating physical data with digital monitoring systems. The benefits of telemedicine have been well known among most of the health subspecialties for many years [2, 3], a well-established practice in every field of chronic pathologies, where solid data of randomized controlled trials and meta-analysis are being published in the last two decades $[4,5]$. These acquisitions led the most important health society to develop guidelines within eHealth support to everyday practice $[2,6]$, with potential several cost-effective benefits [7, 8]. During the COVID-19 outbreak, telehealth has become a widespread practice to ensure access to healthcare in a critical context, in which both physicians and patients share common difficulties in coping with medical assistance.

\footnotetext{
* Address correspondence to this author at Cardiovascular Department, ASST Papa Giovanni XXIII, Bergamo, Italy; Tel: +39 035 2674847;

E-mail: aiacovoni@asst-pg23.it
}

This article describes how telemedicine may be used during the current pandemic, and which measures could be taken into account to improve and sustain telematic medical assistance.

\section{TELEMEDICINE AND TELEHEALTH: USEFUL TOOLS IN HEALTHCARE DELIVERY}

Telehealth is defined by the Centers of Medicare and Medicaid services (CMS) as "the use of telecommunications and information technology to provide access to health assessment, diagnosis, intervention, consultation, supervision, and information across distance" [9]. Delivery of telemedicine can be synchronous, with patient and physicians interacting at the same time (e.g., video-visits), or asynchronous (e.g., reviewing laboratory data). The concept of telemedicine has emerged in recent years and has been used in different healthcare specialties, although it existed since the early twentieth century. Suboptimal systems and structures, as well as scarce regulatory laws have often been depicted as the main reasons of tardive spread of telehealth [10]. During the recent COVID-19 pandemic, necessitating physical distancing, there has been an increased interest and utilization of this tool. Multiple studies and investigations prior to the COVID-19 pandemic have demonstrated the efficacy of telemedicine services. In the cardiovascular setting, a 2017 meta-analysis showed that heart failure patients managed by telemedicine 
systems in addition to standard outpatient visits had lower allcause mortality, lower heart failure admissions, and shorter admission length of stay, compared to in-person approach only [11]. This was attributed to earlier recognition of symptoms and thus prompt intervention. Moreover, a systematic review showed that, in diabetic patients, glycated hemoglobin levels were lower in the telehealth group compared to standard of care [12]. Telemedicine has proven good results also in remote surgery influencing surgical training and practice, allowing monitoring, training or proctoring, thus facing some limitations (e.g., legal and billing issues, cost and network development) [13]. Benefits of telehealth may be linked to improved costeffectiveness, as well as faster access to care with eliminating long distance travel. Cost-effectiveness of telemedicine systems has been widely evaluated and demonstrated in many observational studies [12]: telemedicine services reduced costs related to travel and unnecessary emergency department visits, leading to a better use of resources both by patients and healthcare practitioners.

\section{THE ROLE OF TELEMEDICINE IN THE PANDEMIC ERA}

In this pandemic era, the key role of telehealth is allowing physicians and healthcare professionals to ensure their best clinical assistance by limiting social interaction; in this context, telemedicine should not be considered as an alternative but rather as a support to traditional standard of care. Several paths that connect patients with healthcare workers using telehealth; patients can directly interact with the primary care physician and/or subspecialists through many digital systems, virtual consultations allow reducing the burden on the healthcare system and maintain the precautions during epidemic outbreak. That's why COVID-19 pandemic spread the use of telehealth in most clinical scenarios. Many issues are being experienced by healthcare professionals in delivering the standard clinical assistance: A shortage of human and structural resources, lack of personal protective equipment, lockdown recommendations and financial impact on healthcare economic system are currently the main concerns in the quality standard of patient's care. A recent update from Cardiologic Societies underlines that telemonitoring strategies could help the follow-up of selected high-risk patients in need of close surveillance, by highlighting how remote recording devices could help to preserve personal protective equipment and avoid unnecessary exposure to contagion of healthcare workers [14].

The principles of remote patient management may play the most crucial role in outpatients, ensuring the continuity of care in both COVID-19 and non-COVID-19 pathologies: in this setting, telehealth comprehends several digital experiences which could integrate telephone calls with pieces of information derived by photos, video and medical devices able to monitor vital parameters, such as pulse oxygen saturation or single-lead electrocardiogram (ECG) [15]. Moreover, a variety of virtual platforms have been developed to deliver telehealth to patients via video-conferences, which allow them to maintain direct contact with their general practitioner and/or referral specialists [16]. During the current pandemic, many healthcare centers have expanded the use of telemedicine to continue the follow-up of non-COVID-19 patients [17], thus avoiding coming to the hospital. This appears even more mandatory for the older, frail, and more vulnerable subjects, which represent the majority of the chronic outpatient population. Moreover, telemedicine may be useful to reduce the access to emergency departments and outpatient facilities and to easily consult referral specialists when necessary. Telemedicine and remote monitoring may be useful also in the screening phase of COVID-19 patients; in home-quarantined patients diagnosed with COVID-19, telehealth could support the dramatically increasing demand for care, reducing the burden on primary care practice. During the pandemic, as social distancing became a crucial strategy to minimize virus transmission, the demand for remote visits significantly increased [18]. Sinha et al. [19] evaluated a video visit program at a large, academic primary care in New York, including consecutive adults patients scheduled for video-visits (conducted by attendings, residents, and nurse practitioners) from March 2020 to April 2020. As expected, researchers found that remote visits were a feasible way to take care of both COVID-19 and non-COVID-19 patients, with high satisfaction grades. Although it limited the physician's chances to perform a complete physical examination, the widespread use of home medical devices (i.e. pulse oximeter, automatic arm blood pressure monitor) could partly overcome the gap between digital and physical experience. The usefulness of home medical devices, such as pocket oximeter and smartphone based systems is still a matter of debate, however, several authors recommend their use during pandemic, especially regarding the well-known phenomenon of "silent hypoxia", typical of COVID-19 disease [20]. Moreover, another study conducted on the Veterans Health Administration [21] showed that patients with high clinical and/or social needs were more likely to use the remote and virtual system, while older and/or rural Veterans were less compliant to video visits, highlighting concerns about technical limitations and access difficulties. In this setting, digital education and cheap home medical devices could dramatically enrich the quality of remote visits, leading to useful assessment of clinical status, vital parameters, evaluating the need for a more intensive treatment of sick patients, providing care for subjects in quarantine or recently discharged to home. Meanwhile, in the diagnostic screening phase of exposed or symptomatic subjects, telehealth could help to coordinate testing strategies and triage clinical needs. In this setting, artificial intelligence can be a useful tool in helping physicians, particularly as regards the diagnostic process: Chakraborty et al. described how machine learning and artificial intelligence systems can be used for COVID-19 detecting and diagnosis from X-ray and CT images and it can be a potential method for diagnosis in the radiology department. In addition, artificial intelligence can be used in prediction purposes for COVID-19. Furthermore, it can effectively support drug discovery procedures and can reduce clinical failures [22].

Regarding pharmacotherapy, medical monitoring devices could potentially impact effectiveness and safety of target therapy, tailoring the treatment strategy on patient clinical characteristics. Various drugs, especially hydroxychloroquine and azithromycin, have been used off label in many countries over the world. The main issue regarding the safety profile of these drugs regards its prolonging effects on QT interval [23]; moreover, a combination therapy may have an even worse synergistic effect on QTc prolongation, exposing patients to fatal arrhythmias [24]. For these reasons, a remote ECG and Heart Rate monitoring systems could have been useful in preventing potential adverse events in patients exposed to these 
drugs. Finally, considering the risk of contagion among health care workers in situations other than patient care $[25,26]$, technological implementation could prevent coworker contagion. Indeed, telemedicine systems can be implemented to facilitate consultations between physicians (i.e., between specialists and between specialists and general practitioners), particularly in contexts that would make the exchange of clinical and instrumental information complex (i.e., during the assistance in intensive care units; managing clinical imaging of home monitored patients). Through the implementation of shared servers and virtual platforms with remote access, the eHealth could ensure that several specialists can simultaneously access the clinical and instrumental data of the patient, consult medical records and share specialistic recommendations, minimizing exposure to possible sources of contagion among coworkers and fomites. Telemedicine has proven its usefulness in historical epidemics, including previous coronavirus outbreaks, such as SARS-CoV (coronavirus associated with severe acute respiratory syndrome) and MERS-CoV (Middle East respiratory syndrome coronavirus) [27] by monitoring isolated patients, facilitating eventual sending of medical assistance when aggravating symptoms are identified; in case-tracing, telehealth has just been used in West Africa through a free mobile app that allows patients to send free text messages to receive medical consultation [28]. From October 2014 to May 2015, 6063 requests were registered and 20 suspected cases of Ebola were identified. These studies support the introduction of similar, low-cost systems that could help in the screening phase of COVID-19 pandemic, providing support to infection tracking network. From the home of sick patients to intensive care units (ICU), regarding in-hospital setting telemedicine plays a pivotal role: also in this field it is considered as a consolidated tool that can help solving some critical issues of intensive management, suggesting important implications for future developments. Rosenfeld et al. had already reported a 24-hour remote management of intensive care patients without permanent presence of an on-site physician, using videocall and vital parameters remote monitoring [29]. Moreover, telehealth led to reduction in severity adjusted ICU mortality in the hospital mortality, decreasing the hospitalization length by $34 \%$ with a $33 \%$ cost cutting; finally a concomitant decrease in the incidence of complications has been noticed [29]. In conclusion, in pandemic era eHealth could be widely used from the screening phase to the ICU care in COVID-19 patients as well as in not infected, drastically reducing the risk of contagion, providing a complementary cost effective tool in everyday clinical practice.

\subsection{Limitations}

There are many limits to routine implementation of telemedicine and telehealth technologies, such as inadequate reimbursement, privacy issues, lack of adequate digital infrastructure, incompatible workflow, lack of adequate Internet coverage (given various levels of Internet and servers capacity, some videos may be affected by the poor image quality, lags or dropped calls), restricted financial resources, language barriers, and limited technical skills. Moreover, some telehealth systems are unavailable on a large scale, often requiring private resources and specific training.

In that sense, the COVID-19 pandemic may be an opportunity to invest and promote current technologies into feasible tools for routine clinical practice; we hope these implementations will survive longer than the pandemic.

\section{CONCLUSION}

Telemedicine is transforming the clinical practice during the COVID-19 pandemic and it may be a useful tool to improve clinical assistance and reduce the financial burden on health system in a long-term view. The post-pandemic role of telemedicine is strictly dependent on permanent regulatory solutions; although it cannot adequately replace patientphysician interactions, is imperative to implement this field and make it accessible to the largest part of the population. Longterm strategies may include monitoring high-risk patients by tracking vital signs and other relevant clinical data, make easier the use of teleconsulting so that physicians could safely share specialistic advice in a smarter, quicker way. COVID-19 pandemic may be the catalyst to improve the quality of care and optimize resource utilization with a favorable cost-benefit ratio, ameliorating the patients' quality of life and work conditions of healthcare professionals.

\section{LIST OF ABBREVIATIONS}

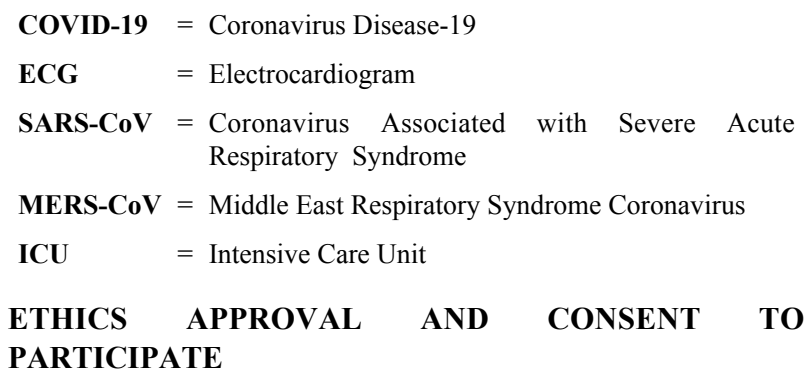

Not applicable.

HUMAN AND ANIMAL RIGHTS

Not applicable.

\section{CONSENT FOR PUBLICATION}

Not applicable.

\section{AVAILABILITY OF DATA AND MATERIALS}

Not applicable.

\section{FUNDING}

None.

\section{CONFLICT OF INTERESTS}

The authors declare no conflict of interest, financial or otherwise.

\section{ACKNOWLEDGEMENTS}

Declared none.

\section{REFERENCES}

[1] N. Lurie, B.G. Carr, B.G. Carr, and S.K. Medical, "The role of telehealth in the medical response to disasters", JAMA Intern. Med., vol. 178 , no. 6 , pp. $745-746,2018$ [http://dx.doi.org/10.1001/jamainternmed.2018.1314] [PMID: 29710200] 
[2] G. Flodgren, A. Rachas, A.J. Farmer, M. Inzitari, and S. Shepperd, "Interactive telemedicine: Effects on professional practice and health care outcomes", Cochrane Database Syst. Rev., no. 9, 2015.CD002098 [http://dx.doi.org/10.1002/14651858.CD002098.pub2] [PMID: 26343551]

[3] S.C. Inglis, R.A. Clark, F.A. McAlister, J. Ball, C. Lewinter, D. Cullington, S. Stewart, and J.G. Cleland, "Structured telephone support or telemonitoring programmes for patients with chronic heart failure", Cochrane Database Syst. Rev., no. 8, 2010.CD007228 [PMID: 20687083]

[4] R. Dierckx, S.C. Inglis, R.A. Clark, D. Prieto-Merino, and J.G.F. Cleland, "Telemedicine in heart failure: New insights from the Cochrane meta-analyses", Eur. J. Heart Fail., vol. 19, no. 3, pp. 304-306, 2017.

[http://dx.doi.org/10.1002/ejhf.759] [PMID: 28251777]

[5] C. Klersy, G. Boriani, A. De Silvestri, G.H. Mairesse, F. Braunschweig, V. Scotti, A. Balduini, M.R. Cowie, and F. Leyva, "Effect of telemonitoring of cardiac implantable electronic devices on healthcare utilization: A meta-analysis of randomized controlled trials in patients with heart failure", Eur. J. Heart Fail., vol. 18, no. 2, pp. 195-204, 2016.

[http://dx.doi.org/10.1002/ejhf.470] [PMID: 26817628]

[6] M.R. Cowie, J. Bax, N. Bruining, J.G. Cleland, F. Koehler, M. Malik, F. Pinto, E. van der Velde, and P. Vardas, "e-Health: A position statement of the European Society of Cardiology", Eur. Heart J., vol. 37 , no. 1 , pp. $63-66,2016$

[http://dx.doi.org/10.1093/eurheartj/ehv416] [PMID: 26303835]

[7] T. Greenhalgh, G. Robert, F. Macfarlane, P. Bate, and O. Kyriakidou, "Diffusion of innovations in service organizations: Systematic review and recommendations", Milbank $Q$., vol. 82, no. 4, pp. 581-629, 2004. [http://dx.doi.org/10.1111/j.0887-378X.2004.00325.x] [PMID: 15595944]

[8] I. de la Torre-Díez, M. López-Coronado, C. Vaca, J.S. Aguado, and C. de Castro, "Cost-utility and cost-effectiveness studies of telemedicine, electronic, and mobile health systems in the literature: A systematic review", Telemed. J. E Health, vol. 21, no. 2, pp. 81-85, 2015. [http://dx.doi.org/10.1089/tmj.2014.0053] [PMID: 25474190]

[9] K.W. Hanson, and H.A. Huskamp, "State health care reform: behavioral health services under Medicaid managed care: The uncertain implications of state variation", Psychiatr. Serv., vol. 52, no. 4, pp. 447-450, 2001

[http://dx.doi.org/10.1176/appi.ps.52.4.447] [PMID: 11274487]

[10] C. Scott Kruse, P. Karem, K. Shifflett, L. Vegi, K. Ravi, and M. Brooks, "Evaluating barriers to adopting telemedicine worldwide: A systematic review", J. Telemed. Telecare, vol. 24, no. 1, pp. 4-12, 2018 .

[http://dx.doi.org/10.1177/1357633X16674087] [PMID: 29320966]

[11] M.H. Lin, W.L. Yuan, T.C. Huang, H.F. Zhang, J.T. Mai, and J.F. Wang, "Clinical effectiveness of telemedicine for chronic heart failure: A systematic review and meta-analysis", J. Investig. Med., vol. 65, no. 5, pp. 899-911, 2017.

[http://dx.doi.org/10.1136/jim-2016-000199] [PMID: 28330835]

[12] R.E. Powell, J.M. Henstenburg, G. Cooper, J.E. Hollander, and K.L. Rising, "Patient perceptions of telehealth primary care video visits", Ann. Fam. Med., vol. 15, no. 3, pp. 225-229, 2017.

[http://dx.doi.org/10.1370/afm.2095] [PMID: 28483887]

[13] A. Mohan, U.U. Wara, M.T. Arshad Shaikh, R.M. Rahman, and Z.A. Zaidi, "Telesurgery and robotics: An improved and efficient era", Cureus, vol. 13, no. 3, 2021.e14124

[http://dx.doi.org/10.7759/cureus.14124] [PMID: 33927932]

[14] N. Varma, N.F. Marrouche, L. Aguinaga, C.M. Albert, E. Arbelo, J.I. Choi, M.K. Chung, G. Conte, L. Dagher, L.M. Epstein, H. Ghanbari, J.K. Han, H. Heidbuchel, H. Huang, D.R. Lakkireddy, T. Ngarmukos, A.M. Russo, E.B. Saad, L.C. Saenz Morales, K.E. Sandau, A.R.M. Sridhar, E.C. Stecker, and P.D. Varosy, "HRS/EHRA/APHRS/LAHRS/ACC/AHA worldwide practice update for telehealth and arrhythmia monitoring during and after a pandemic", Europace, vol. 23, no. 2, p. 313, 2021.

[http://dx.doi.org/10.1093/europace/euaa187] [PMID: 32526011]
[15] N. Varma, and R.P. Ricci, "Telemedicine and cardiac implants: What is the benefit?", Eur. Heart J., vol. 34, no. 25, pp. 1885-1895, 2013. [http://dx.doi.org/10.1093/eurheartj/ehs388] [PMID: 23211231]

[16] NHSX Information Governance. Available at: https://www.nhsx.nhs.uk/information-governance/

[17] P. Webster, "Virtual health care in the era of COVID-19", Lancet, vol. 395 , no. 10231 , pp. $1180-1181,2020$.

[http://dx.doi.org/10.1016/S0140-6736(20)30818-7]

[PMID: 32278374

[18] A. Mehrotra, K. Ray, D.M. Brockmeyer, M.L. Barnett, and J.A. Bender, "Rapidly converting to "Virtual Practices": Outpatient care in the era of COVID-19", N Engl J Med Catalyst, no. April, 2020. [http://dx.doi.org/10.1056/CAT.20.0091]

[19] S. Sinha, L.M. Kern, L.F. Gingras, E. Reshetnyak, J. Tung, F. Pelzman, T.A. McGrath, and M.R. Sterling, "Implementation of video visits during COVID-19: Lessons learned from a primary care practice in new york city", Front. Public Health, vol. 8, p. 514, 2020 [http://dx.doi.org/10.3389/fpubh.2020.00514] [PMID: 33042950

[20] A.M. Luks, and E.R. Swenson, "Pulse oximetry for monitoring patients with COVID-19 at home. Potential pitfalls and practical guidance", Ann. Am. Thorac. Soc., vol. 17, no. 9, pp. 1040-1046, 2020. [http://dx.doi.org/10.1513/AnnalsATS.202005-418FR] [PMID: 32521167]

[21] J.M. Ferguson, J. Jacobs, M. Yefimova, L. Greene, L. Heyworth, and D.M. Zulman, "Virtual care expansion in the Veterans Health Administration during the COVID-19 pandemic: Clinical services and patient characteristics associated with utilization", J. Am. Med. Inform. Assoc., vol. 28, no. 3, pp. 453-462, 2021.

[http://dx.doi.org/10.1093/jamia/ocaa284] [PMID: 33125032]

[22] C. Chakraborty, and A.N. Abougreen, "Intelligent internet of things and advanced machine learning techniques for COVID-19", EAI Endorsed Trans. Pervasive Health Technol., vol. 7, no. 26, pp. 1-14, 2021.

[http://dx.doi.org/10.4108/eai.28-1-2021.168505]

[23] I.L. Haeusler, X.H.S. Chan, P.J. Guérin, and N.J. White, "The arrhythmogenic cardiotoxicity of the quinoline and structurally related antimalarial drugs: A systematic review", BMC Med., vol. 16, no. 1, p. $200,2018$.

[http://dx.doi.org/10.1186/s12916-018-1188-2] [PMID: 30400791]

[24] T. Fiolet, A. Guihur, M.E. Rebeaud, M. Mulot, N. Peiffer-Smadja, and Y. Mahamat-Saleh, "Effect of hydroxychloroquine with or without azithromycin on the mortality of coronavirus disease 2019 (COVID-19) patients: A systematic review and meta-analysis", Clin. Microbiol. Infect., vol. 27, no. 1, pp. 19-27, 2021.

[http://dx.doi.org/10.1016/j.cmi.2020.08.022] [PMID: 32860962]

[25] C.R. MacIntyre, and A.A. Chughtai, "A rapid systematic review of the efficacy of face masks and respirators against coronaviruses and other respiratory transmissible viruses for the community, healthcare workers and sick patients", Int. J. Nurs. Stud., vol. 108, 2020.103629 [http://dx.doi.org/10.1016/j.ijnurstu.2020.103629] [PMID: 32512240]

[26] J.J. Bartoszko, M.A.M. Farooqi, W. Alhazzani, M. Loeb, and M. Loeb, "Medical masks vs N95 respirators for preventing COVID-19 in healthcare workers: A systematic review and meta-analysis of randomized trials", Influenza Other Respir. Viruses, vol. 14, no. 4, pp. 365-373, 2020.

[http://dx.doi.org/10.1111/irv.12745] [PMID: 32246890]

[27] R Ohannessian, "Telemedicine: Potential applications in epidemic situations", Eur Res Telemedicine/La Rech Eur en Télémédecine, vol. 4, no. 3, pp. 95-98.

[http://dx.doi.org/10.1016/j.eurtel.2015.08.002]

[28] Doctor Gratis, Accessed online, 2015. Available from: http://www.doctorgratis.org

[29] B.A. Rosenfeld, T. Dorman, M.J. Breslow, P. Pronovost, M. Jenckes, N. Zhang, G. Anderson, and H. Rubin, "Intensive care unit telemedicine: Alternate paradigm for providing continuous intensivist care", Crit. Care Med., vol. 28, no. 12, pp. 3925-3931, 2000. [http://dx.doi.org/10.1097/00003246-200012000-00034] [PMID: 11153637] 